\title{
Empirical verification of heterogeneous DNA fragments generated from wheat genome-specific SSR primers
}

\author{
Qijiao Chen ${ }^{1,2}$, Lianquan Zhang ${ }^{1,2}$, Zhongwei Yuan ${ }^{1,2}$, Zehong Yan ${ }^{1,2}$, Youliang Zheng ${ }^{1,2}$, \\ Genlou Sun ${ }^{3}$, and Dengcai Liu'1,2,4
}

${ }^{1}$ Triticeae Research Institute, Sichuan Agricultural University, Dujiangyan, Sichuan 611830, China; ${ }^{2}$ Key Laboratory of Crop Genetic Resources and Improvement, Ministry of Education, Sichuan Agricultural University, Yaan, Sichuan 625014, China; and ${ }^{3}$ Department of Biology, Saint Mary's University, Halifax, Nova Scotia, Canada B3H 3C3. Received 16 March 2008, accepted 14 July 2008.

\begin{abstract}
Chen, Q.-J., Zhang, L., Yuan, Z., Yan, Z., Zheng, Y., Sun, G. and Liu, D. 2008. Empirical verification of heterogeneous DNA fragments generated from wheat genome-specific SSR primers. Can. J. Plant Sci. 88: 1065-1071. Due to the high polymorphisms between synthetic hexaploid wheat (SHW) and common wheat, SHW has been widely used in genetic studies. The transferability of simple sequence repeats (SSR) among common wheat and its donor species, Triticum turgidum and Aegilops tauschii, and their SHW suggested the possibility that some SSRs, specific for a single locus in common wheat, might appear in two or more loci in SHWs. This is an important genetic issue when using synthetic hexaploid wheat population and SSR for mapping. However, it is largely ignored and never empirically well verified. The present study addressed this issue by using the well-studied SSR marker Xgwm261 as an example. The Xgwm261 produced a 192 bp fragment specific to chromosome 2D in common wheat Chinese Spring, but generated a 176 bp fragment in the D genome of Ae. tauschii AS60. Chromosomal location and DNA sequence data revealed that the176 bp fragment also donated by 2B chromosome of durum wheat Langdon. These results indicated that although a single $176 \mathrm{bp}$ fragment was appeared in synthetic hexaploid wheat Syn-SAU-5 between Langdon and AS60, the fragment contained two different loci, one from chromosome 2D of AS60 and the other from 2B of Langdon which were confirmed by the segregating analysis of SSR Xgwm261 in 185 plants from a F 2 population between Syn-SAU-5 and Chinese Spring. If Xgwm261 in Syn-SAU-5 was considered as a single locus in genetic analysis, distorted segregation or incorrect conclusions would be yielded. A proposed strategy to avoid this problem is to include SHW's parental T. turgidum and Ae. tauschii in SSR analysis as control for polymorphism detection.
\end{abstract}

Key words: Synthetic hexaploid wheat, microsatellite, segregation distortion, Xgwm261, transferability

Chen, Q.-J., Zhang, L., Yuan, Z., Yan, Z., Zheng, Y., Sun, G. et Liu, D. 2008. Vérification empirique des fragments d'ADN hétérogènes issus des amorces SSR spécifiques au blé. Can. J. Plant Sci. 88: 1065-1071. La génétique recourt largement au blé hexaploïde synthétique (BHS) à cause de son grand polymorphisme avec le blé commun. Le fait qu'on puisse transférer les SSR du blé commun et des espèces donatrices Triticum turgidum et Aegilops tauschii au BHS laisse croire que certains SSR, spécifiques à un locus donné du blé commun, se retrouvent sur deux locus ou plus du BHS. Il s'agit d'un point important à tenir compte en génétique, lorsqu'on utilise le BHS et des SSR pour cartographier le génome. Pourtant, on le fait rarement et la chose n'a jamais été vérifiée de manière empirique. L'étude devait combler cette lacune en prenant le marqueur SSR bien connu Xgwm261 comme exemple. Le marqueur Xgwm261 produit un fragment de 192 bp spécifique au chromosome 2D du blé commun Chinese Spring et un fragment de $176 \mathrm{bp}$ du génome D d'Ae. tauschii AS60. L'emplacement du marqueur sur le chromosome et les données sur la séquence de l'ADN révèlent que le fragment de 176 bp vient aussi du chromosome 2B du blé dur Langdon. On en conclut que le fragment de 176 bp qui apparaît dans le BHS Syn-SAU-5, entre Langdon et AS60, comprend deux locus, le premier du chromosome 2D de AS60 et le second du chromosome 2B de Langdon, ce que confirme l'analyse par ségrégation du SSR Xgwm261 chez 185 plants de la $\mathrm{F}_{2}$ d'un croisement entre Syn-SAU-5 et Chinese Spring. Si le SSR Xgwm261 de Syn-SAU-5 était considéré comme émanant d'un locus unique dans une analyse génétique, il en ressortirait des conclusions erronées. Les auteurs proposent une façon d'éviter le problème, en l'occurrence inclure les parents T. turgidum et Ae. tauschii du BHS comme témoins dans l'analyse des SSR pour déceler le polymorphisme.

Mots clés: Blé hexaploïde synthétique (BHS), microsatellite (SSR), distorsion par ségrégation, Xgwm261, transférabilité

Qi-Jiao Chen and Lian-Quan Zhang have contributed equally to this research.

${ }^{4}$ To whom correspondence should be addressed (e-mail: dcliu7@yahoo.com)
Abbreviations: CS, Chinese Spring; LDN, Langdon; QTL, quantitative trait loci; SDL, segregation-distortion loci; SHW, synthetic hexaploid wheat; SSR, simple sequence repeats 
Bread or common wheat (Triticum aestivum L., $2 n=$ $6 x=42$, AABBDD), one of the most remarkable allopolyploids, was formed by spontaneous hexaploidization after the intercrossing between $T$. turgidum and Ae. tauschii (Kihara 1944; McFadden and Sears 1944). Limited genotypes of donor species were involved in the origin of common wheat. Due to the evolution bottleneck, numerous genetic variations in the ancestral $T$. turgidum or Ae. tauschii are not represented at the hexaploid level (Reif et al. 2005; Warburton et al. 2006). The creation of synthetic hexaploid wheats (SHW) by chromosome doubling of $T$. turgidum $\times$ Ae. tauschii hybrids (McFadden and Sears 1944), which is analogous to the origin of hexaploid wheat, has been presenting a promising method to unlock the diversity conserved in the progenitors of wheat (Hoisington et al. 1999; Van Ginkel and Ogbonnaya 2007). In addition to their usefulness in breeding, due to the high polymorphisms in SHWs, they have been widely used to construct populations for genetic analysis.

Microsatellite or simple sequence repeat (SSR) markers are widely used for genetic studies and breeding programs in common wheat. The molecular genetic maps of common wheat contain over 1500 SSRs, some of them were mapped to single locus and specific to individual chromosome or chromosome arm (Bryan et al. 1997; Röder et al. 1998, 2004; Pestsova et al. 2000; Somers et al. 2004). SSRs from common wheat have been shown to give amplification in many related or alien species and vice versa, which is known as "transferability" (Pestova et al. 2000; Sourdille et al. 2001; Guyomarc'h et al. 2002a,b; Sharma et al. 2002; Kuleung et al. 2004; Adonina et al. 2005; Zhang et al. 2004, 2005, 2007a). Comparison of SSR patterns among newly synthesized hexaploid wheat (SHW) and its donor T. turgidum and Ae. tauschii indicated that some SSR markers located on the D genome of common wheat could amplify the SSR sequences from both the A/B genome of T. turgidum and D genome of Ae. tauschii. The amplified fragments were also detected in SHWs. These results suggested that these SSR markers specific to $\mathrm{D}$ genome in common wheat were probably nonspecific to D genome in SHWs. Similarly, some SSR primers specific to $\mathrm{A} / \mathrm{B}$ genome in common wheat were probably nonspecific to the A/B genome in SHWs (Zhang et al. 2004, 2005, 2007a). Given that the wheat genome is so large and complex, it can be hypothesized that the chance of amplifying non-specific sequences is very high. This hypothesis needs to be verified by more effective methods, such as DNA sequencing and chromosome location analysis of the transferable fragments.

The well-studied SSR Xgwm261, a (CT/GA)n marker in common wheat (Röder et al. 1998) is of particular interest to breeders because of its association with plant height. The Xgwm261 on the short arm of chromosome $2 \mathrm{D}$ is linked to the Rht8 with a genetic distance of 0.6 cM between them (Korzun et al. 1998; Röder et al. 1998). At locus Xgwm261, besides a number of rare alleles, alleles of 204, 192, 174, and 164 bp were presented in most varieties of common wheat (Worland et al. 1998, 2001; Chebotar et al. 2001; Ahmad and Sorrells 2002; Manifesto and Suárez 2002; Liu et al. 2005; Ellis et al. 2007). In our study, a set of welldesigned experiments clearly indicated that Xgwm261, specific for a singe locus on 2DS in common wheat, appeared in two loci in a SHW.

\section{MATERIALS AND METHODS}

\section{Plant Materials}

The durum wheat (T. turgidum ssp. durum) cv. Langdon (LDN) and its disomic substitution line 2D (2B), in which a pair of chromosome $2 \mathrm{~B}$ in cv. Langdon were replaced by a pair of homoeologous chromosome 2D of common wheat Chinese Spring, were developed by Joppa and Williams (1983). The Langdon lines were provided by Dr. L. R. Joppa, Fargo, ND, North Dakota State University, USA. Ae. tauschii accession AS60 with desirable crossability (Liu et al. 2002) was used for crossing with Langdon lines. Common wheat lines Chinese Spring (CS) and Chinese Spring nullisomic 2B-tetrasomic 2D (CS N2BT2D) were obtained from E. R. Sears, University of Missouri, Columbia, MO. CS N2BT2D has four copies of chromosome 2D and has zero copies of chromosome $2 \mathrm{~B}$.

\section{Production and Cytological Observation of Synthetic Wheat}

The durum wheat lines Langdon and Langdon 2D(2B) as female parents were crossed with Ae. tauschii (accession AS60). The emasculation and pollination techniques described by Liu et al. (1999) were followed. No embryo rescue technique or hormone treatments were applied when producing the $F_{1}$ hybrids. $F_{1}$ seeds were germinated in Petri dishes before transplanting into the field. Spikes of the $F_{1}$ and $F_{2}$ hybrid plants were randomly selected for further investigation. These selected spikes were bagged before flowering to prevent cross-contamination.

Somatic chromosomes were counted using squashed root-tip preparations (Zhang et al. 2007b). Root tips (1-3 cm long) were cut from the seedlings and pretreated in ice water at $0-4^{\circ} \mathrm{C}$ for $20-24 \mathrm{~h}$, then fixed in Carnoy's fixative I (three parts of $95 \%$ ethanol to one part of glacial acetic acid) overnight. Feulgen staining was applied to the fixed root tips to visualize chromosomes. The $\mathrm{F}_{2}$ hybrid plants with $2 n=42$ chromosomes were selected. These plants were grown $10 \mathrm{~cm}$ apart with $60-\mathrm{cm}$ row spacing in the experimental field of Triticeae Research Institute of Sichuan Agricultural University at Dujiangyan City, Sichuan Province, China.

\section{SSR Analysis}

Total genomic DNA was isolated from young fresh leaves using $2 \times$ CTAB method according to Zhang et al. (2004). The SSR Xgwm261 primer sequences were: 
5'-CTCCCTGTACGCCTAAGGC- $3^{\prime}$ and 5'-CTCGCG CTACTAGCCATTG-3' (Röder et al. 1998). PCR reaction contains: 1 unit ExTaq polymerase (TaKaRa, Dalian, China), $1 \times$ ExTaq buffer $\left(\mathrm{Mg}^{2+}\right.$ free $), 1.5 \mathrm{mM}$ $\mathrm{MgCl}_{2}, 0.2 \mathrm{mM}$ dNTPs, $200 \mathrm{nM}$ of each primer, and 50 $100 \mathrm{ng}$ template DNA in $20 \mu \mathrm{L}$ final volumes. Thermocycling conditions were: initial denaturation at $94^{\circ} \mathrm{C}$ for $5 \mathrm{~min} ; 38$ cycles of $30 \mathrm{~s}$ at $94^{\circ} \mathrm{C}, 30 \mathrm{~s}$ at $55^{\circ} \mathrm{C}, 30 \mathrm{~s}$ at $72^{\circ} \mathrm{C}$; and a finial extension at $72^{\circ} \mathrm{C}$ for $5 \mathrm{~min}$. PCR was performed in a MJ Research thermocycler (Watertown MA). The amplified fragments were separated by electrophoresis in a denaturing $6 \%$ polyacrylamide gel and visualized with the silver-staining method (Zhang et al. 2004).

\section{Cloning and Sequence Analysis}

The target fragments were cut from the polyacrylamide gel and boiled in $20 \mu \mathrm{L} \mathrm{ddH}_{2} \mathrm{O}$ for $5 \mathrm{~min}$ and then centrifuged at $4000 \times g$ for $10 \mathrm{~min}$. The upper liquid was used to conduct the second PCR reaction using the same program as above. The final Xgwm261 PCR products were separated on $3.0 \%$ agarose gels and the expected fragments were purified from gels using E.Z.N.A. ${ }^{\circledR} \mathrm{Gel}$ Extraction Kit (OMIGA, USA.). The purified PCR products were cloned into pMD18-T vector using cloning kit from TaKaRa Biotechnology (Dalian) Co. Five positive clones from each plant materials were identified, and commercially sequenced by the SunBiotech company (Beijing, China). Sequence alignments were conducted using the DNAMAN 6.0 Demo software (Lynnon Biosoft).

\section{Genetic Analysis on $\mathbf{F}_{\mathbf{2}}$ Population}

The $F_{2}$ population was developed from the cross between common wheat Chinese Spring and new synthetic hexaploid wheat Syn-SAU-5. Randomly chosen $185 \mathrm{~F}_{2}$ individuals were analyzed using marker Xgwm261. SSR bands on silver staining gels were calculated based on the band size. The examined plants were classified into three groups: (I) with 176 bp band, (II) with 192 bp band, (III) with both 176 bp and 192 bp bands. Chi-square analysis was performed with SPSS software version 11.0 (SPSS Inc., Chicago, IL) to assess the goodness-of-fit of marker Xgwm261 between observed and expected segregation ratio in the segregating population. A $P$ value less than 0.01 was regarded as highly significant.

\section{RESULTS \\ Production of Synthetic Hexaploid Wheat, Nullisomic 2B-tetrasomic 2D}

In 2004, LDN and LDN 2D(2B) were pollinated with Ae. tauschii accession AS60. Triploid $\mathrm{F}_{1}$ seeds were obtained from both crosses without embryo rescue. Averaged crossability was $3.16 \%$ for the LDN $\times$ AS60 and $0.87 \%$ for the LDN $2 \mathrm{D}(2 \mathrm{~B}) \times \mathrm{AS} 60$. Most of the $\mathrm{F}_{1}$ seeds were germinated and produced vigorously growing
$F_{1}$ plants. As expected, the $F_{1}$ hybrid plants had 21 chromosomes, and showed tough tenacious brown glumes, which were obviously inherited from Ae. tauschii. All the triploid $\mathrm{F}_{1}$ hybrids were highly fertile and gave high seed set. In 2005, the seed set rate was $36.87 \%$ (755 seeds from 2048 florets) for hybrids of LDN-Ae. tauschii and 65.32\% (290 seeds from 444 florets) for the $\mathrm{F}_{1}$ hybrids of LDN 2D(2B)-Ae. tauschii.

Chromosome numbers were checked for 34 randomly selected $\mathrm{F}_{2}$ seeds of LDN-Ae. tauschii. Twenty-nine had 42 chromosomes. Ten out of eleven LDN 2D(2B)-Ae. tauschii $\mathrm{F}_{2}$ seeds had 42 chromosomes. The high frequency of selfed seed set rate and $F_{2}$ seeds with 42 chromosomes indicated that unreduced gametes in the LDN lines $\times$ AS60 hybrids are common. Previous cytological observations on male gametogenesis have shown that meiotic restitution is responsible for the production of functional unreduced male gametes, resulting in spontaneously amphidiploids with high sefled seedsettings in LDN-Ae. tauschii hybrids, which is controlled by the gene from LDN (Xu and Joppa 1995, 2000; Jauhar et al. 2000; Jauhar 2003; Matsuoka and Nasuda 2004).

Newly SHW lines with $2 n=42$, derived from LDN $\times$ Ae. tauschii, were obtained. Of which, the SHW line Syn-SAU-5 was further crossed with common wheat Chinese Spring to produce $\mathrm{F}_{2}$ population. SHW lines with $2 n=42$ chromosomes from LDN 2D(2B)-Ae. tauschii should be nullisomic 2B-tetrasomic 2D, which has four copies of chromosome 2D, two from Chinese Spring and two from Ae. tauschii line AS60, and has zero copies of chromosome 2B. The synthetic nullisomic 2B-tetrasomic 2D is abbreviated as Syn-SAU N2BT2D.

\section{SSR Analysis of the Newly Synthetic Wheats and Their Parents}

Common wheat cultivar Chinese Spring (CS) has a 192 bp Xgwm261 allele (Worland et al. 2001) which was also observed in CS in this study (Fig. 1), but absent in CS N2DT2B. This result indicated that SSR Xgwm261 was only located on chromosome $2 \mathrm{D}$, and specific to chromosome 2D in CS.

As expected, one PCR fragment of SSR Xgwm261was detected in Ae. tauschii line AS60 (Fig. 1) with D genome. It is interesting that the same fragment of $A e$. tauschii AS60 appeared in tetraploid wheat LDN (AABB genomes). However, the fragment size was smaller than that in common wheat CS. This transferable PCR fragment in LDN was not detected in LDN $2 \mathrm{D}(2 \mathrm{~B})$ in which a pair of chromosome $2 \mathrm{~B}$ were replaced by a pair of chromosome $2 \mathrm{D}$ of common wheat CS. A 192 bp allele was observed in the 2D of CS (Fig. 1). This result indicated that the PCR fragment in LDN was located on chromosome 2B.

In some cases, the fragment can be amplified through template-primer mismatch and/or modified PCR conditions, resulting in nonspecific amplification. To verify amplification specificity, each five clones from $\mathrm{Xgwm} 261$ 


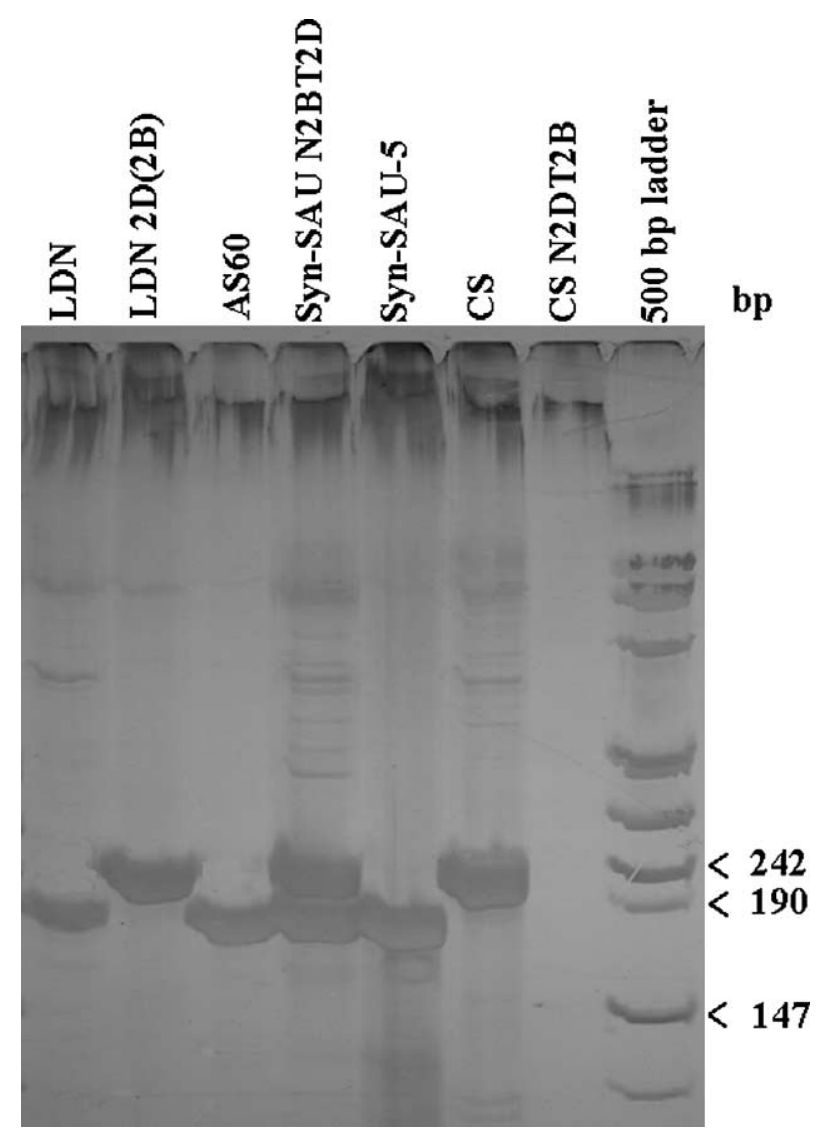

Fig. 1. The amplified products for SSR Xgwm261 from durum LDN, LDN 2D(2B), Ae. tauschii AS60 and their synthetic hexaploid wheats Syn-SAU-5, Syn-SAU N2BT2D and common wheat CS and CS N2BT2D, revealed by $6 \%$ denaturing polyacrylamide gels.

amplified LDN and Ae. tauschii AS60 product were sequenced. Sequence alignment revealed that all the clones had the same sequences with a length of $176 \mathrm{bp}$ (Fig. 2). Comparison between the $176 \mathrm{bp}$ sequence and $192 \mathrm{bp}$ sequence (provided by Liu et al. 2005) indicated that the $176 \mathrm{bp}$ alleles is composed of $\left[(\mathrm{CT})_{11} \mathrm{AG}\right]$ repeat motif, and the 192 bp alleles is composed of [(CT) $\left.)_{21}\right]$ repeat motif (Fig. 2).

The synthetic nullisomic 2B-tetrasomic 2D, Syn-SAU N2BT2D showed two distinct bands, a 192 bp from chromosome 2D of common wheat CS, a $176 \mathrm{bp}$ from 2D of Ae. tauschii. The synthetic hexaploid wheat SynSAU-5 between LDN and Ae. tauschii displayed the 176 bp band, which probably contains two different loci, one for chromosomes $2 \mathrm{~B}$ of LDN and the other for 2D of Ae. tauschii (Fig. 1). However, since the fragment size of $2 \mathrm{D}$ in Ae. tauschii and 2B in LDN is same, they can not be distinguished in synthetic wheat Syn-SAU-5 background.

\section{SSR Analysis on $F_{2}$ Segregation Population Between Syn-SAU-5 and CS}

SSR analysis on $F_{2}$ segregation population verified that SSR Xgwm261 in Syn-SAU-5 contained two different loci. CS and Syn-SAU-5 produced two polymorphic bands, with a length of $192 \mathrm{bp}$ and $176 \mathrm{bp}$ in Xgwm261 loci, respectively. All the $\mathrm{F}_{1}$ hybrids between CS and Syn-SAU-5 showed both bands. Their $F_{2}$ generation exhibited three phenotypes, i.e., one band with $176 \mathrm{bp}$, two bands with 176 and $192 \mathrm{bp}$, and one band with 192 bp. Theoretically, if SSR Xgwm261 with $176 \mathrm{bp}$ is a single locus in Syn-SAU-5, its $\mathrm{F}_{2}$ hybrids with CS would show a ratio of 1 (176 bp): 2 (176 bp and $192 \mathrm{bp}$ ): 1 (192 bp). However, if the Xgwm261 contains two loci in different chromosomes, $2 \mathrm{~B}$ and $2 \mathrm{D}, \mathrm{F}_{2}$ hybrids would result in a ratio of 4 (176 bp): 11 (176 bp and $192 \mathrm{bp}): 1$ (192 bp) (Fig. 3). Among the $185 \mathrm{~F}_{2}$ plants from populations between Syn-SAU-5 and CS, 46, 125 and 14 plants showed the $176 \mathrm{bp}, 176 \mathrm{bp}+192 \mathrm{bp}$ and 192 bp bands, respectively. Chi-square test revealed that Xgwm261 was segregated at a ratio of $4(176 \mathrm{bp}): 11$ (176 bp and 192 bp): 1 (192 bp), which was in agreement with the expected segregation of two loci, one co-dominant locus on 2D and one dominant locus on $2 \mathrm{~B}$, rather than single locus at 0.01 significant level (Table 1).

\section{DISCUSSION}

Identification of markers linked to Mendelian and quantitative trait loci (QTL) of agronomic importance has been achieved using co-segregation and QTL analyses, and linked markers are being used in marker-assisted selection programs. Reliable genetic maps are essential for these purposes. However, segregation distortion, deviations from expected Mendelian segregation ratios, which is a common biological phenomenon and occurs in a number of species, can affect the accuracy of genetic linkage maps (Lyttle 1991; Lorieux et al. 1995a,b; Foisset and Delourme 1996; Cloutier et al. 1997; Zhu et al. 2007). Extremely distorted segregation could result in a wrong linkage group. Low-magnitude segregation distortion may not affect the detection of proper linkage. However, it can affect the estimation of map distances and the order of markers.

There are many possible causes for segregation distortion (Lyttle 1991; Foisset and Delourme 1996). One of them may result from a selection process during gametogenesis, fertilization or germination caused by segregation-distortion loci (SDL). If segregation distortion is caused by SDL, all markers in the vicinity of the SDL will be affected and result in a cluster with distorted ratios. Errors in marker genotyping may also cause systematic deviations from expected segregation ratio. However, this could lead to mis-scoring of only 
LDN-261(2B) CTCCCTGTACGCCTAAGGCCTTGTACA AAGCAACGTCCTTAAAAAATAAATTAGATTTTCTTTAG AS60-261(2D) CTCCCTGTACGCCTAAGGCCTTGTACA AAGCAACGTGCTTAAAAAATAAATTAGATTTTCTTTAG
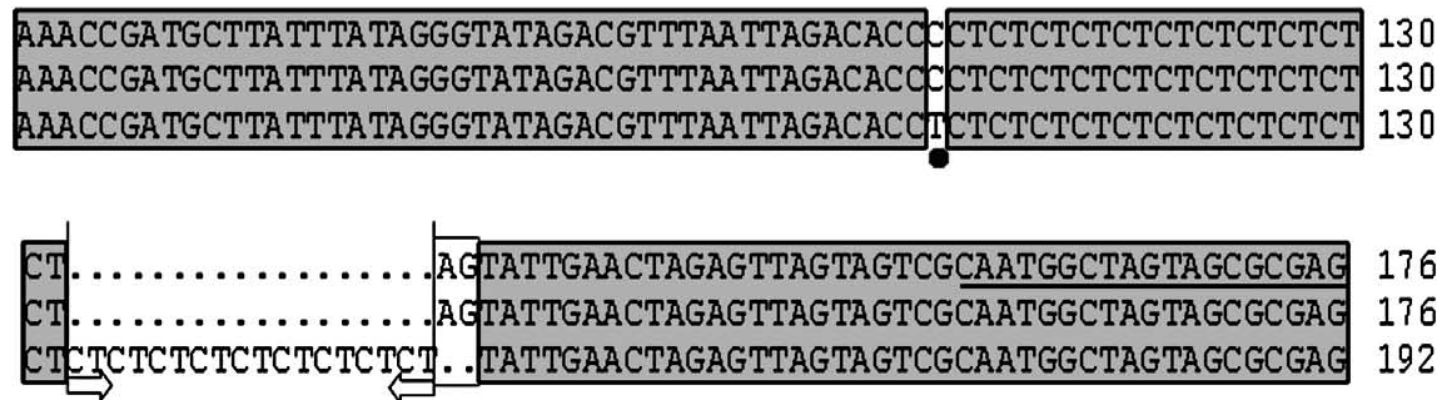

Fig. 2. Sequence comparison of SSR Xgwm261 among the alleles from chromosomes 2D in Ae. tauschii AS60 and common wheat CS and 2B in durum wheat LDN. Primer sequences were underlined; bold circles represent the nucleotide replacements; arrow heads showed the dinucleotide (CT) motif insertions/deletions; The AG insertion/deletion were boxed.

a single marker. SSR bands could have been mis-scored and given the wrong allelic designation. The discriminative ability of gel-separation technology can affect accuracy and reproducibility of band scoring. "Stutter" bands, which are especially prone to occur in di-repeat SSRs, can also cause incorrect genetic scoring of bands (Smith et al. 1997).

The present study demonstrated that wrong locus determination may be an alterative cause for segregation distortion. According to the data of the chromosomal location and DNA sequence, two Xgwm261 loci, each existed in chromosomes 2B and 2D of Syn-SAU-5, respectively. In the $F_{2}$ segregation population of SynSAU-5 $\times$ CS,$X g w m 261$ bands were segregated into a 4:11:1 ratio in agreement with the segregation of two loci, one co-dominant locus on $2 \mathrm{D}$ and one dominant locus on 2B. However, since the two loci in Syn-SAU-5 have the same size, they could not be distinguished from each other in the SSR analyses. According to classic analysis procedure on SSR, Xgwm261 would be misscored as single locus with the 1:2:1 ratio in genetic analyses. As a consequence, the segregation ratio for

$$
\begin{array}{rlll}
\text { Parental generation: } 2 B^{176} 2 B^{176} 2 D^{176} 2 D^{176} & X & 2 B^{\text {null }} 2 B^{\text {null }} 2 D^{192} 2 D^{192} \\
\text { (Synthetic wheat Syn-SAU-5) } & \downarrow & \text { (Chinese Spring) }
\end{array}
$$

$F_{1}$ generation:

$2 B^{176} 2 B^{\text {null }} 2 D^{176} 2 D^{192}$

\begin{tabular}{|c|c|c|c|c|}
\hline & $2 \mathrm{~B}^{176} 2 \mathrm{D}^{176}$ & $2 \mathrm{~B}^{176} 2 \mathrm{D}^{192^{-}}$ & $2 \mathrm{~B}^{\text {null }} 2 \mathrm{D}^{176}$ & $2 \mathrm{~B}^{\text {null }} 2 \mathrm{D}^{192}$ \\
\hline $\begin{array}{c}\text { Male gametes } \\
22 B^{176} 2 D^{176}\end{array}$ & 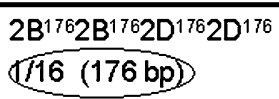 & $\begin{array}{l}2 B^{176} 2 B^{176} D^{176} 2 D^{192} \\
1 / 16(176+192 b p)\end{array}$ & $\begin{array}{l}2 \mathrm{~B}^{176} 2 \mathrm{~B}^{\mathrm{nu}} 2 \mathrm{D}^{176} \mathrm{D}^{176} \\
1 / 16(176 \mathrm{bP})\end{array}$ & $\begin{array}{l}2 B^{176} 2 B^{\text {null } 2 D^{176} 2 D^{192}} \\
1 / 16(176+192 b p)\end{array}$ \\
\hline $2 B^{176} 2 D^{192}$ & $\begin{array}{l}2 B^{176} B^{176} D^{192} 2 D^{176} \\
1 / 16(176+192 b p)\end{array}$ & $\begin{array}{l}2 B^{176} 2 B^{176} D^{192} 2 D^{192} \\
1 / 16(176+192 b p)\end{array}$ & $\begin{array}{l}2 \mathrm{~B}^{176} 2 \mathrm{~B}^{\text {null }} 2 \mathrm{D}^{1922 \mathrm{D}^{176}} \\
1 / 16(176+192 \mathrm{bp})\end{array}$ & $\begin{array}{l}2 B^{176} \mathrm{~B}^{\text {null}} 2 \mathrm{D}^{192} 2 \mathrm{D}^{192} \\
1 / 16(176+192 \mathrm{bp})\end{array}$ \\
\hline $2 \mathrm{~B}^{\text {null }} 2 \mathrm{D}^{176}$ & $\begin{array}{l}2 \mathrm{~B}^{\text {null }} 2 \mathrm{~B}^{176} 2 \mathrm{D}^{176} \mathrm{D}^{1766} \\
\text { (1716 (176 bP) }\end{array}$ & $\begin{array}{l}2 \mathrm{~B}^{\text {null }} 2 \mathrm{~B}^{176} \mathrm{D}^{176} \mathrm{CD}^{192} \\
1 / 16(176+192 \mathrm{bp})\end{array}$ & 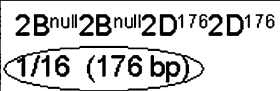 & $\begin{array}{l}2 B^{\text {null }} 2 B^{\text {null }} 2 D^{176} 2^{192} \\
1 / 16(176+192 b p)\end{array}$ \\
\hline $2 \mathrm{~B}^{\text {null }} 2 \mathrm{D}^{192}$ & $\begin{array}{l}2 B^{\text {null }} 2 B^{176} 2 D^{192} 2 D^{176} \\
1 / 16(176+192 b p)\end{array}$ & $\begin{array}{l}2 B^{\text {null }} 2 B^{176} 2 D^{192} 2 D^{192} \\
1 / 16(176+192 b p)\end{array}$ & 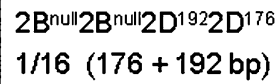 & 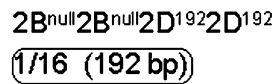 \\
\hline
\end{tabular}

$F_{2}$ generation:

Fig. 3. The Punnett square of the SSR genotypes and phenotypes in the hybrid of Syn-SAU $-5 \times$ Chinese Spring. 


\begin{tabular}{lccc}
\hline Table 1. Chi-square analysis for $\mathbf{F}_{\mathbf{2}}$ segregation population of Syn-SAU-5 $\times$ Chinese Spring & \\
\hline Genotypes & $\begin{array}{c}\text { No. of } \\
\text { observed plants }\end{array}$ & $\begin{array}{c}\text { No. of expected } \\
\text { plants for two loci }(4: 11: 1)\end{array}$ & $\begin{array}{c}\text { No. of expected } \\
\text { plants for single locus }(1: 2: 1)\end{array}$ \\
\hline $176 \mathrm{bp}$ & 46 & 46.25 & 46.25 \\
$176 \mathrm{bp}+192 \mathrm{bp}$ & 125 & 127.19 & 92.50 \\
$192 \mathrm{bp}$ & 14 & 11.56 & 46.25 \\
& & $\chi^{2}=0.554$ & $\chi^{2}=33.91^{* *}$ \\
\hline
\end{tabular}

$\chi^{2} 0.01,2=9.21$

Xgwm261 would be distorted with a higher frequency for heterozygous (176 bp+192 bp) and lower for CS (192 bp). Although only $F_{2}$ segregation populations were analyzed in the present study, distorted segregations would be produced in other populations, such as DH populations and recombined inbred lines if we considered two loci as single locus.

This study only demonstrated an example for two loci by using well-studied SSR Xgwm261. Previous studies have indicated that many SSRs are transferable from common wheat to its donor species, T. turgidum and Ae. tauschii, and their SHWs (Pestova et al. 2000; Sourdille et al. 2001; Guyomarc'h et al. 2002a,b; Sharma et al. 2002; Zhang et al. 2004, 2005, 2007a), which suggested the possibility that some SSRs, specific for a singe locus in common wheat, could have two, three or more loci in SHWs.

In summary, our data suggest that caution should be used in analyzing the genetic population between common wheat and SHW. As example of Xgwm261, in genetic mapping, those transferable SSRs could be mis-scored as single locus, and would yield inaccurate information. To avoid the problems from this type of SSR markers, an effective method is to use the corresponding parents of SHWs, T. turgidum and Ae. tauschii, as controls in polymorphism analysis of SSR. The SSRs that failed to reveal the differences between D-genome of Ae. tauschii and A/B genomes of $T$. turgidum should not be selected in further analysis.

\section{ACKNOWLEDGMENTS}

This work is supported by the projects of New Century Excellent Talents in University (No. NCET-04-0908) and Changjiang Scholars and Innovative Research Team in University (No. IRT0453) of Chinese Ministry of Education; National Natural Science Foundation of China (No. 30700495); Education Department, Science and technology Department of Sichuan province.

Adonina, I. G., Salina, E. A., Pestsova, E. G. and Röder, M. S. 2005. Transferability of wheat microsatellites to diploid Aegilops species and determination of chromosomal localizations of microsatellites in the S genome. Genome 48: 959-970. Ahmad, M. and Sorrells, M. E. 2002. Distribution of microsatellite alleles linked to Rht8 dwarfing gene in wheat. Euphytica 123: 235-240.

Bryan, G. J., Collins, A. J., Stephenson, P., Orry, A., Smith, J. B. and Gale, M. D. 1997. Isolation and characterization of microsatellites from hexaploid bread wheat. Theor. Appl. Genet. 94: 557-563.

Cloutier, S., Cappadocia, M. and Landry, B. S. 1997. Analysis of RFLP mapping inaccuracy in Brassica napus L. Theor. Appl. Genet. 95: 83-91.

Chebotar, S. V., Korzun, V. N. and Sibolap, Yu. M. 2001. Allele distribution at locus WMS261 marking the dwarfing gene Rht8 in common wheat cultivars of Southern Ukraine. Russ. J. Genet. 37: 894-898.

Ellis, M. H., Bonnett, D. G. and Rebetzke, G. J. 2007. A $192 \mathrm{bp}$ allele at the $X g w m 261$ locus is not always associated with the Rht 8 dwarfing gene in wheat (Triticum aestivum L.). Euphytica 157: 209-214.

Foisset, N. and Delourme, R. 1996. Segregation distortions in androgenic plants. Pages 189-201 in S. M. Jain, S. K. Sopory, and R. E. Veilleux, eds. In vitro haploid production in higher plants, Vol. 2. Kluwer Academic Publishers, Dordrecht, the Netherlands.

Guyomarc'h, H., Sourdille, P., Charmet, G., Edwards, K. J. and Bernard, M. 2002a. Characterization of polymorphic microsatellite markers from Aegilops tauschii and transferability to the D-genome of bread wheat. Theor. Appl. Genet. 104: 1164 1172.

Guyomarc'h, H., Sourdille, P., Edwards, K. J. and Bernard, M. 2002b. Studies of the transferability of microsatellites derived from Triticum tauschii to hexaploid wheat and to diploid related species using amplification, hybridization and sequence comparisons. Theor. Appl. Genet. 105: 736-744.

Hoisington, D., Khairallah, M., Reeves, T., Ribaut, J. M., Skovmand, B., Taba, S. and Warburton, M. 1999. Plant genetic resources: What can they contribute toward increased crop productivity? Proc. Natl. Acad. Sci. USA 96: 5937-5943.

Jauhar, P. P., Dogramaci-Altuntepe, M., Peterson, T. S. and Almouslem, A. B. 2000. Seedset on synthetic haploids of durum wheat: cytological and molecular investigations. Crop Sci. 40: $1742-1749$.

Jauhar, P. P. 2003. Formation of $2 n$ gametes in durum wheat haploids: sexual polyploidization. Euphytica 133: 81-94.

Joppa, L. R. and Williams, N. D. 1983. The Langdon durum disomic-substitutions: development, characteristics and uses. Agron. Abstr. 68.

Kihara, H. 1944. Discovery of the DD-analyser, one of the ancestors of Triticum vulgare. Agric. Hortic. 19: 889-890.

Kuleung, C., Baenziger, P. S. and Dweikat, I. 2004. Transferability of SSR markers among wheat, rye, and triticale. Theor. Appl. Genet. 108: 1147-1150.

Korzun, V., Röder, M. S., Ganal, M. W., Worland, A. J. and Law, C. N. 1998. Genetic analysis of the dwarfing gene (Rht8) in wheat. Part I. Molecular mapping of Rht 8 on the short arm of chromosome 2D of bread wheat (Triticum aestivum L.) Theor. Appl. Genet. 96: 1104-1109. 
Liu, D. C., Yen, C., Yang, J. L., Zheng, Y. L. and Lan, X. J. 1999. The chromosomal distribution of crossability genes in tetraploid wheat Triticum turgidum L. cv. Ailanmai native to Sichuan, China. Euphytica 108: 79-82.

Liu, D. C., Lan, X. J., Yang, Z. J., Zheng, Y. L., Wei, Y. M. and Zhou, Y. H. 2002. A unique Aegilops tauschii genotype needless to immature embryo culture in cross with wheat. Acta Bot. Sin. 44: 708-713.

Liu, Y., Liu, D. C., Zhang, H. Y., Wang, J., Sun, J. Z., Guo, X. L. and Zhang, A. M. 2005. Allelic variation, sequence determination and microsatellite screening at the XGWM261 locus in Chinese hexaploid wheat (Triticum aestivum) varieties. Euphytica 145: 103-112.

Lorieux, M., Goffinet, B., Perrier, X., León. D. G. D. and Lanaud, C. 1995a. Maximum likelihood models for mapping genetic markers showing segregation distortion. 1. Backcross population. Theor. Appl. Genet. 90: 73-80.

Lorieux, M., Perrier, X., Goffinet, B., Lanaud, C. and León. D. G. D. 1995b. Maximum likelihood models for mapping genetic markers showing segregation distortion. 2. F2 population. Theor. Appl. Genet. 90: 81-89.

Lyttle, T. W. 1991. Segregation distorters. Annu. Rev. Genet. 25: 511-557.

Manifesto, M. M. and Suárez, E. Y. 2002. Microsatellite screening of the Rht 8 dwarfing gene in Argentinian wheat cultivars. Annual Wheat Newsletter 48: 23-24.

Matsuoka, Y. and Nasuda, S. 2004. Durum wheat as a candidate for the unknown female progenitor of bread wheat: an empirical study with a highly fertile $\mathrm{F}_{1}$ hybrid with Aegilops tauschii Coss. Theor. Appl. Genet. 109: 1710-1717.

McFadden, E. S. and Sears, E. R. 1944. The artificial synthesis of Triticum spleta. Rec. Genet. Soc. Am. 13: 26-27.

Pestsova, E. G., Ganal, M. W. and Röder, M. S. 2000. Isolation and mapping of microsatellite markers specific for the D genome of bread wheat. Genome 43: 689-697.

Reif, J. C., Zhang, P., Dreisigacker, S., Warburton, M. L., Van Ginkel, M., Hoisington, D., Bohn, M. and Melchinger, A. E. 2005. Wheat genetic diversity trends during domestication and breeding. Theor. Appl. Genet. 110: 859-864.

Röder, M. S., Korzun, V., Wendehake, K., Plaschke, J., Tixier, M. H., Leroy, P. and Ganal, M. W. 1998. A microsatellite map in wheat. Genetics 149: 2007-2023.

Röder, M. S., Huang, X. Q. and Ganal, M. W. 2004. Wheat microsatellites in plant breeding -potential and implications. Pages 255-266 in H. Loertz and G. Wenzel, eds. Molecular markers in plant breeding. Springer-Verlag, Germany.

Sharma, S., Balyan, H. S., Kulwal, P. L., Kumar, N., Varshney, R. K., Prasad, M. and Gupta, P. K. 2002. Study of interspecific SSR polymorphism among 14 species from Triticum-Aegilops group. Wheat Inf. Serv. 95: 23-28.

Smith, J. S. C., Chin, E. C. L., Shu, H., Smith, O. S., Wall, S. J., Senior, M. L., Mitchell, S. E., Kresovich, S. and Ziegle, J. 1997. An evaluation of the utility of SSR loci as molecular markers in maize (Zey mays L.): comparisons with data from RFLPs and pedigree. Theor. Appl. Genet. 95: 163-173.

Somers, D. J., Isaac, P. and Edwards, K. 2004. A high-density microsatellite consensus map for bread wheat (Triticum aestivum L.). Theor. Appl. Genet. 109: 1432-2242.

Sourdille, P., Tavaud, M., Charmet, G. and Bernard, M. 2001. Transferability of wheat microsatellites to diploid Triticeae species carrying the A, B and D genomes. Theor. Appl. Genet. 103: $346-352$.

Van Ginkel, M. and Ogbonnaya, F. 2007. Novel genetic diversity from synthetic wheats in breeding cultivars for changing production conditions. Field Crops Res. 104: 86-94. Warburton, M. L., Crossa, J., Franco, J., Mujeeb-Kazi, A., Trethowan, R., Rajaram, S., Pfeiffer, W., Zhang, P., Dreisigacker, S. and Van Ginkel, M. 2006. Bringing wild relatives back into the family: recovering genetic diversity in CIMMYT improved wheat germplasm. Euphytica 149: 289-301.

Worland, A. J., Korzun, V., Röder, M. S., Ganal, M. W. and Law, C. N. 1998. Genetic analysis of the dwarfing gene Rht8 in wheat. Part II. The distribution and adaptive significance of allelic variants at the Rht8 locus of wheat as revealed by microsatellite screening. Theor. Appl. Genet. 96: 1110-1120.

Worland, A. J., Sayers, E. J. and Korzun, V. 2001. Allelic variation at the dwarfing gene Rht 8 locus and its significance in international breeding programmes. Euphytica 119: 155-159.

Xu, S. J. and Joppa, L. R. 1995. Mechanisms and inheritance of first division restitution in hybrids of wheat, rye, and Aegilops squarrosa. Genome 38: 607-615.

Xu, S. J. and Joppa, L. R. 2000. First-division restitution in hybrids of Langdon durum disomic substitution lines with rye and Aegilops squarrosa. Plant Breed. 119: 233-241.

Zhang, L. Q., Liu, D. C., Yan, Z. H., Lan, X. J., Zheng, Y. L. and Zhou, Y. H. 2004. Rapid changes of microsatellite flanking sequence in the allopolyploidization of new synthesized hexaploid wheat. Sci. China C Life Sci. 47: 553-561.

Zhang, L. Q., Sun, G. L., Yan, Z. H., Chen, Q. J., Yuan, Z. W., Lan, X. J., Zheng, Y. L. and Liu, D. C. 2007a. Comparison of newly synthetic hexaploid wheat with its donors on SSR products. J. Genet. Genom. 34: 939-946.

Zhang, L. Q., Yen, Y., Zheng, Y. L. and Liu, D.C. 2007b. Meiotic restriction in emmer wheat is controlled by one or more nuclear genes that continue to function in derived lines. Sex. Plant Reprod. 20: 159-166.

Zhang, P. Z., Dreisigacker, S., Melchinger, A. E., Reif, J. C., Mujeeb-Kazi, A., Van Ginkel, M., Hoisington, D. and Warburton, M. L. 2005. Quantifying novel sequence variation and selective advantage in synthetic hexaploid wheats and their backcross-derived lines using SSR markers. Mol. Breed. 15: $1-10$.

Zhu, C. S., Wang, F. H., Wang, J. F., Li, G. J., Zhang, H. S. and Zhang, Y. M. 2007. Reconstruction of linkage maps in the distorted segregation populations of backcross, doubled haploid and recombinant inbred lines. Chin. Sci. Bull. 52: 16481653. 